\title{
Left ventricular remodeling with preserved function after coronary microembolization: the effect of methylprednisolone
}

\author{
Jianying Ma ${ }^{1,2}$, Juying Qian ${ }^{1,2}$, Shufu Chang ${ }^{1,2}$, Zhangwei Chen ${ }^{1,2}$, Hang Jin ${ }^{3}$, Mengsu Zeng ${ }^{3}$, Yunzeng Zou ${ }^{1,2}$
} and Junbo $\mathrm{Ge}^{1,2^{*}}$

\begin{abstract}
Background: The objective of this study was to evaluate changes in left ventricular ejection fraction (LVEF) and left ventricular remodeling after coronary microembolization (CME) and to investigate the protective effects of methylprednisolone (MTP).

Methods: CME was induced by injection of microspheres ( $42 \mu \mathrm{m}$ Dynospheres) into left anterior descending artery of mini swine. The animals were divided into two groups. Group $1(n=9)$ received 120,000 microspheres and Group 2 $(n=7)$ received 120,000 microspheres following intravenous administration of $30 \mathrm{mg} / \mathrm{kg}$ MTP. Contrast-enhanced magnetic resonance imaging (CeMRI) was performed at baseline, $6 \mathrm{~h}$ after intervention, and 1 week later.

Results: In Group 1, LVEF was significantly decreased at $6 \mathrm{~h}$ but recovered 1 week. This was accompanied by continuing left ventricular remodeling. In Group 2, LVEF remained unchanged at all assessment times. LVEF measured at $6 \mathrm{~h}$ and 1 week after CME in Group 1 and Group 2 was $0.39 \pm 0.06$ and $0.44 \pm 0.04$, and $0.44 \pm 0.04$ and $0.48 \pm 0.03$, respectively (Both $P>0.05$ ). Hyperenchancement at the anterior wall of the left ventricle was shown by MRI at $6 \mathrm{~h}$ in Group 1 but not in Group 2. The hyperenhanced area in Group 1 was $7.77 \pm 1.49 \%$ of left ventricular mass.
\end{abstract}

Conclusions: The consequence of CME is left ventricular dilation with preserved LVEF. Pretreatment with MTP appears to have a cardioprotective effect on left ventricular remodeling.

Keywords: Coronary artery, Microembolization, Magnetic resonance imaging, Left ventricular ejection fraction, Methylprednisolone

\section{Background}

Coronary microembolization (CME) can potentially lead to a perfusion-contraction mismatch, inflammation, and regional myocardial contractile dysfunction in the absence of coronary artery stenosis [1-7]. It is well recognized that left ventricular ejection fraction (LVEF) decreases during the acute phase following CME and recovers 1 week later $[4,8,9]$. However, previous studies with contrast-enhanced magnetic resonance imaging (CeMRI) have shown that microinfarction and left ventricular remodeling are well defined consequences of CME [8-11]. Assessment of left

\footnotetext{
* Correspondence: ge.junbo@zs-hospital.sh.cn

'Department of Cardiology, Zhongshan Hospital, Fudan University, Shanghai 200032, China

${ }^{2}$ Shanghai Institute of Cardiovascular Diseases, Shanghai 200032, China

Full list of author information is available at the end of the article
}

ventricular function using MRI is generally considered to be highly accurate and the current gold standard for calculating ventricular volumetric parameters $[12,13]$.

Glucocorticoids have been used in various diseases to prevent or minimize inflammation [14], but controversy exists concerning the use of glucocorticoids during acute myocardial infarction [15]. To date only one study has assessed the effect of glucocorticoids after microembolization [4]. The study suggested that progressive myocardial dysfunction can be prevented by intravenous injection of methylprednisolone (MTP) prior to the onset of CME [4]. The researchers proposed that MTP decreased the inflammatory reaction caused by microinfarction [4]. In the present study, we further assessed changes in left ventricular function after CME and the effect of MTP pretreated

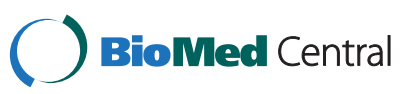

(c) 2014 Ma et al.; licensee BioMed Central Ltd. This is an open access article distributed under the terms of the Creative Commons Attribution License (http://creativecommons.org/licenses/by/2.0), which permits unrestricted use, distribution, and reproduction in any medium, provided the original work is properly cited. 
before CME using CeMRI in an animal model developed by our team.

\section{Methods \\ CME methodology}

Sixteen mini swine of either sex (20 to $25 \mathrm{~kg}$ body weight) were sedated with intramuscular ketamine (5 to $10 \mathrm{mg} / \mathrm{kg}$ ) and diazepam (5 to $10 \mathrm{mg} / \mathrm{kg}$ ). Anesthesia was maintained with $3 \%$ pentobarbital sodium intravenously $(3 \mathrm{mg} / \mathrm{kg} / \mathrm{h}$ ) until the experiment was completed.

The right femoral artery and vein were dissected and $7 \mathrm{~F}$ and $6 \mathrm{~F}$ vascular sheaths were placed in each vessel, respectively. Prior to coronary cannulation, animals were anticoagulated with intravenous heparin (5,000 IU bolus followed by $100 \mathrm{IU} / \mathrm{kg} / \mathrm{h})$. For coronary angiography, a $6 \mathrm{~F}$ $\mathrm{XB} 3.5$ guiding catheter was used for the left coronary artery system via the femoral approach. A $2.8 \mathrm{~F}$ infusion catheter (Cordis Inc., Johnson \& Johnson) was then placed in the left anterior descending (LAD) artery between the second and third diagonal branches. Microembolization was induced by continuous injection of 120,000 microspheres (42 $\mu \mathrm{m}$ Dynospheres; Dyno Particles; Lillestrøm, Norway) into the LAD followed by a $6 \mathrm{~mL}$ saline flush. Animals in Group $2(n=7)$ received $30 \mathrm{mg} / \mathrm{kg}$ MTP (in $10 \mathrm{~mL} 0.9 \% \mathrm{NaCl}$ solution) injected intravenously $30 \mathrm{~min}$ before preparation for surgery. Animals in Group $1(n=9)$ received no MTP.

Systemic hemodynamics parameters and myocardial function were measured at baseline, $6 \mathrm{~h}$, and 1 week after CME. Aortic pressure was monitored through the guiding catheter.

All animals were observed until they were awake and breathing spontaneously without intubation. The swine were anesthetized a second time 1 week later.

The experimental protocol was approved by the Animal Care and Use Committee of Fudan University, China. The animals were handled according to the guidelines of the American Physiological Society.

\section{Methods for MRI}

MRI was performed at baseline, $6 \mathrm{~h}$, and 1 week after CME using 1.5 T Magnetom (Erlangen, Siemens AG, Germany). Transverse, two-chamber, and four-chamber left ventricular long-axis scout images were obtained to determine the final short-axis image plane. Cine MR images were acquired in contiguous short-axis planes from the apex to the base of the heart to measure left ventricular function. After the cine MR images were obtained, the animals received an intravenous bolus of $0.20 \mathrm{mmol} / \mathrm{kg}$ Gd-DTPA (Magnevist; Schering, Berlin, German) at a rate of $4 \mathrm{~mL} / \mathrm{s}$ by means of an infusion pump. This was followed by a $10 \mathrm{~mL}$ flush of saline at a rate of $4 \mathrm{~mL} / \mathrm{s}$. After $10 \mathrm{~min}$, delayed images were acquired using a Turbo_flash_T1WI_PSIR_segmented sequence.
The inversion time was determined at $10 \mathrm{~min}$ with TIScout sequence. Results were analyzed at Siemens Syngo Leonardo workstation [8].

\section{Statistical analysis}

Statistical analysis was undertaken using SPSS version 11.0 software. Data were presented as means and standard deviations $( \pm \mathrm{SD})$. Within group changes in hemodynamics (left ventricular ejection fraction (LVEF), left ventricular end diastolic volume (LVEDV), and left ventricular end systolic volume (LVESV)) were compared using a general linear model of variance for repeated measures. Independent $t$ test were used to compare LVEF, LVEDV, and LVESV between groups. Values of $P<0.05$ were considered statistically significant.

\section{Results}

Coronary thrombolysis in myocardial infarction (TIMI) grade 3 coronary flow was maintained immediately after microembolization and hemodynamic parameters remained stable before and after CME.

In Group 1, LVEF decreased at $6 \mathrm{~h}(P<0.01)$ and returned to baseline 1 week after CME. In addition LVEDV and LVESV were higher 1 week than at baseline in this group $(P<0.01)$. In Group 2, there was no significant change in LVEF, LVEDV, and LVESV before or after CME. There was no difference in LVEF, LVEDV, or LVESV at $6 \mathrm{~h}$ between Group 1 and Group 2 ( $P=0.08$ for LVEF). However, at 1 week before and after changes in LVEDV and LVESV were significantly lower in Group 2 than in Group $1(P<0.01)$. Before and after changes in LVEF at $6 \mathrm{~h}$ were significantly lower in Group 2 than in Group $1(P<0.01)$. The results are shown in Table 1 and in Figures 1 and 2.

Hyperenchancement at anterior wall of left ventricle was apparent on CeMRI $6 \mathrm{~h}$ after CME in Group 1 but

Table 1 Changes in LVEF, LVEDV, and LVESV in Groups 1 and 2 before and after CME

\begin{tabular}{llll}
\hline & Baseline & $\mathbf{6} \boldsymbol{h}$ & One week \\
\hline LVEF & & & \\
Group 1 & $0.48 \pm 0.06$ & $0.39 \pm 0.06^{\mathrm{a}}$ & $0.46 \pm 0.05$ \\
Group 2 & $0.47 \pm 0.04$ & $0.44 \pm 0.04$ & $0.48 \pm 0.03$ \\
LVEDV (mL) & & & \\
Group 1 & $40.4 \pm 3.1$ & $40.8 \pm 3.0$ & $48.5 \pm 4.0^{\mathrm{a}}$ \\
Group 2 & $38.0 \pm 4.7$ & $39.0 \pm 6.6$ & $37.4 \pm 5.4$ \\
LVESV (mL) & & & \\
Group 1 & $21.0 \pm 1.9$ & $24.7 \pm 1.8$ & $26.2 \pm 3.2^{\mathrm{a}}$ \\
Group 2 & $20.2 \pm 2.4$ & $21.8 \pm 3.4$ & $19.4 \pm 2.3$
\end{tabular}

Group 1: coronary microembolization with 120,000 microspheres Group 2: coronary microembolization with 120,000 microspheres and pretreatment with methylprednisolone.

LVEF: left ventricular ejection fraction; LVEDV: left ventricular end dilatolic volume; LVESV: left ventricular systolic volume.

${ }^{a}$ Compared with baseline $P<0.01$. 


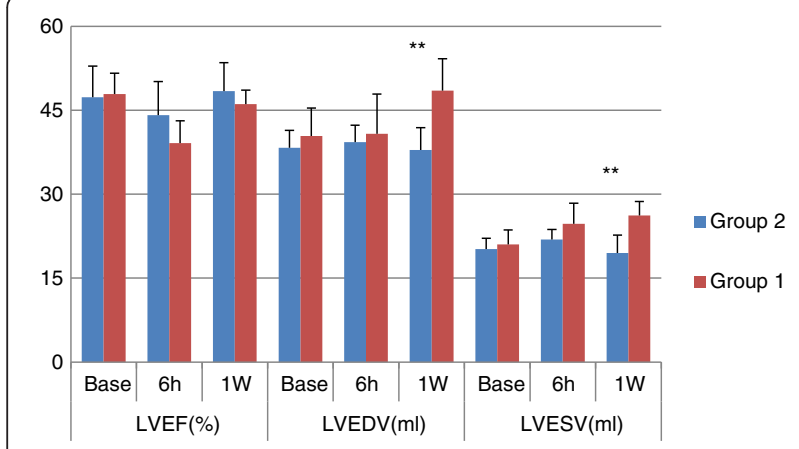

Figure 1 Comparsion of LVEF, LVEDV, and LVESV between Groups 1 and 2. ${ }^{* *}$ Compared with Group 1, $P<0.01$. Group 1: coronary microembolization with 120,000 microspheres. Group 2: coronary microembolization with 120,000 microspheres and pretreatment with methylprednisolone. LVEF: left ventricular ejection fraction; LVEDV: left ventricular end diastolic volume. LVESV: left ventricular end systolic volume.

not in Group 2. The hyperenhanced zone was clearly visible at the anterior myocardium from the papillary to apical level (Figure 3). The area of hyperenhancment in Group 1 was $7.77 \pm 1.49 \%$ of the left ventricular mass.

\section{Discussion}

CME is a frequent phenomenon in patients with coronary heart disease and is particularly prevalent in patients with acute coronary syndrome (ACS). It is a marker for fatal events, especially in patients undergoing percutaneous coronary intervention (PCI) [16-19]. Rupture of the atherosclerotic plaque in the epicardial coronary artery is the main cause of CME in clinical practice [18]. The consequence of CME may also be related fatal events in patients with unstable angina pectoris $[17,18]$.

In the present study, we addressed changes in left ventricular function after $\mathrm{CME}$ and investigated the possible protective role of methylprednisolone. Our objective was to better understand the development of CME and to evaluate the possible benefit of glucocorticoid pretreatment. Using an experimental model developed by our team, we demonstrated impairment of left ventricular function (evidenced by changes in LVEF) at $6 \mathrm{~h}$, recovers spontaneously 1 week later after CME, but left ventricular remodeling continues [8]. We also showed that pretreatment with MTP before CME may prevent the progression of remodeling.

Based on these findings, the observed changes were thought to be related to an inflammatory response rather than to micro-infarction after CME. Previous studies have demonstrated that myocardial infarction following CME affects only about $2 \%$ to $5 \%$ of the perfusion territory, and that apoptosis is almost negligible [1,5]. Our results supported these findings as hyperenhancement $6 \mathrm{~h}$ after CME accounted for no more that $8 \%$ of left ventricular mass.

Previous studies have shown that inflammatory responses induced by microinfarction, is mediated by monocytes, macrophage infiltration, and the release of inflammatory factors $[2,8]$. In a previous study we showed that the degree of inflammation was markedly higher in the embolized zone than that in the control zone [9]. We also demonstrated that increased serum MCP-1 promotes leukocyte infiltration and that this is followed by a classical inflammatory response [9], suggesting that inflammation and oxygen-free radicals are involved in left ventricular remodeling [5,9,20-23].

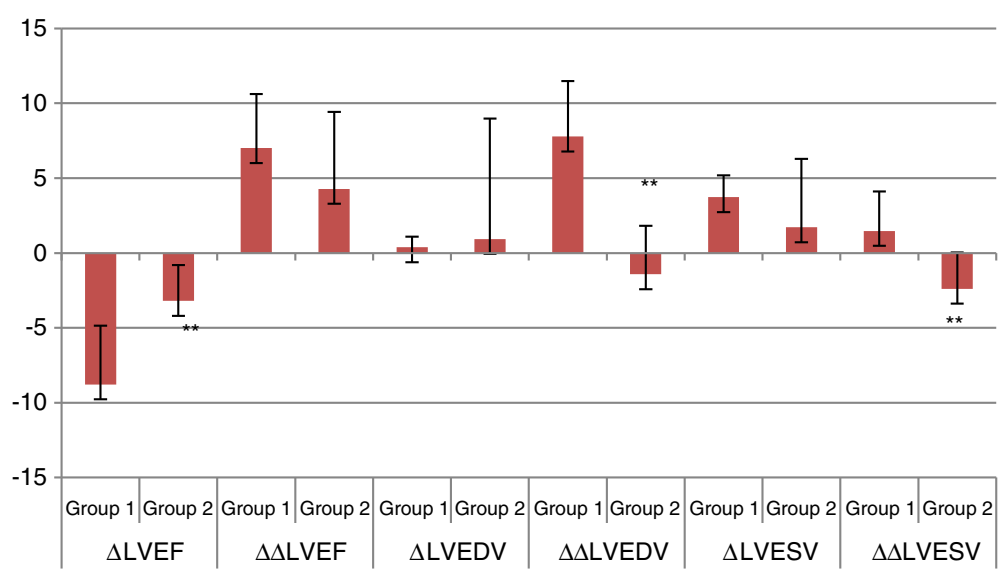

Figure 2 Before and after changes in left ventricular function between Groups 1 and 2. ${ }^{* *}$ Compared with Group 1, P<0.01. Group 1: coronary microembolization with 120,000 microspheres. Group 2: coronary microembolization with 120,000 microspheres and pretreatment with methylprednisolone. ( $\Delta$ LVEF $=6 \mathrm{~h}$ LVEF-baseline LVEF, $\Delta \Delta$ LVEF $=1$ week LVEF-baseline LVEF; $\Delta$ LVEDV $=6$ h LVEDV-baseline LVEDV, $\Delta \Delta$ LVEDV $=1$ week LVEDV-baseline LVEDV; $\Delta$ LVESV $=6$ h LVESV-baseline LVESV, $\Delta \Delta$ LVESV = 1 week LVESV-baseline LVESV. LVEF: left ventricular ejection fraction; LVEDV: left ventricular end diastolic volume. LVESV: left ventricular end systolic volume. Before and after changes in LVEF at $6 \mathrm{~h}$ were significantly lower in Group 2 than in Group 1. At 1 week before and after changes in LVEDV and LVESV were significantly lower in Group 2 than in Group 1. 


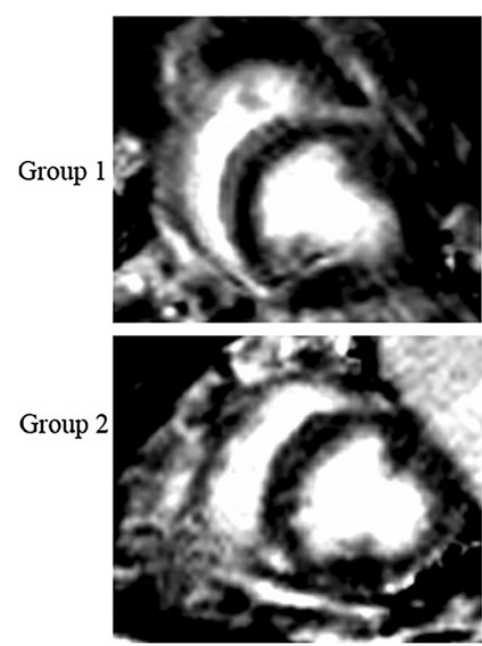

Baseline

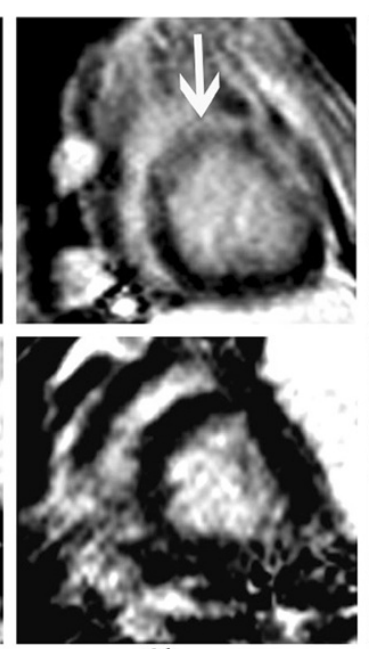

$6 \mathrm{~h}$

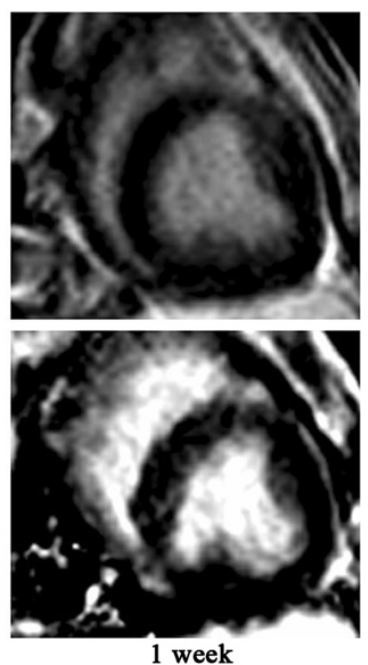

1 week

Figure 3 Changes in CeMRI before and after CME in Group 1 and Group 2. Group 1: coronary microembolization with 120,000 microspheres. Group 2: coronary microembolization with 120,000 microspheres and pretreatment with methylprednisolone. Hyperenhancement was demonstrated at $6 \mathrm{~h}$ following CME in Group 1 (white arrow) but not in Group 2. One week after CME there was no evidence of hyperenhancement on CeMRI in Group 1.

In our experiments LVEF was preserved 1 week after CME due to left ventricular remodeling, which occurred as a consequence of the persistent inflammatory response in the embolized zone. A previous study, using MRI to evaluate changes after CME, demonstrated a significant decline in LVEF $1 \mathrm{~h}$ and 1 week after intervention [11]. The difference between these findings and our own may be related to differences in the sizes of microspheres used in the animal models. Thus, it is possible that larger diameter microspheres might result in a more persistent decline in LVEF.

In our previous study, hyperenchancement was observed $6 \mathrm{~h}$ after CME [8]. This hyperenhancement was disappeared 1 week later without any treatment. The reason for this may be related to the presence of acute myocardial microinfarction and acute myocardial edema $6 \mathrm{~h}$ after CME. However, after 1 week, the myocardial edema would have disappeared and the spatial resolution of MRI may not have been high enough to detect the experimentally induced microinfarcts.

An important finding of the present study was that pretreatment with MTP eliminated the hyperenhancement at $6 \mathrm{~h}$ after CME and protected the decline of LVEF as well as left ventricular remodeling. The anti-inflammatory effects of glucocorticoids have been widely used for the treatment of a variety of diseases in clinical practice. In experimental studies, glucocorticoids have been shown to attenuate the interaction of leukocytes with endothelium [24] and to inhibit the infiltration of macrophages/ monocytes [25]. Glucocorticoid-induced suppression of the generation and release of inflammatory cytokines and mediators have also been demonstrated $[25,26]$. However, the effects of glucocorticoids in the treatment of acute myocardial infarction remained controversial [27].

A previous study reported that MTP was able to protect against progressive myocardial contractile dysfunction after CME, suggested that it may have a cardioprotective effect in this setting [4]. These findings are supported by the results of the present study and suggest that pretreatment with MTP inhibits the inflammatory response and attenuates myocardial edema induced by CME [4]. This effect was also reflected by the absence of delayed hyperenhancement $6 \mathrm{~h}$ after CME in the pretreated group. As a result of the reduced levels of the inflammation, LVEF remained unchanged and left ventricular remodeling was no longer present 1 week after CME. These results suggest that pretreatment with glucocorticoids may prevent left ventricular remodeling after CME in the clinical practice [4]. However further studies are needed to substantiate these findings.

As there was no control group in our study it was not possible to eliminate changes due to the surgical procedure. However, previous studies have demonstrated this type of animal model closely resembles CME seen in clinical practice and in previous studies control groups were not used. A second limitation is that this animal model resembles but does not exactly replicate the sequence of events that occurs following complications of percutaneous coronary intervention. In clinical practice the thromboemboli are frequently much larger, are of non-uniform diameter, and do not always result in myocardial damage. In future studies, a longer observation period should be adopted to observe the long-term effects of CME and glucorcoticoids on left ventricular remodeling. 


\section{Conclusions}

Our results demonstrate that experimentally induced CME resulted in left ventricular dilation with preserved LVEF. Pretreatment with MTP had a cardioprotective effect on left ventricular remodeling. These findings are of potential significance for the clinical management of CME.

\begin{abstract}
Abbreviations
ACS: Acute coronary syndrome; CeMRI: Contrast enhanced magnetic resonance imaging; CME: Coronary microembolization; LAD: Left anterior descending; LVEDV: Left ventricular end diastolic volume; LVEF: Left ventricular ejection fraction; LVESV: Left ventricular end systolic volume; MTP: Methylprednisolone; PCl: Percutaneous coronary intervention; TIMI: Thrombolysis in myocardial infarction.
\end{abstract}

\section{Competing interests}

The authors have no competing interests.

\section{Authors' contributions}

JM carried out the animal studies, performed the statistical analysis, and drafted the manuscript. JQ participated in the design of the study and helped to draft the manuscript. SC participated in the animal model preparation and construction. ZC participated in the animal model construction. HJ participated in the MRI and data collection. MZ participated in the MRI. YZ participated in the design of the study and helped to perform the statistical analysis. JG conceived of the study, and participated in its design and helped to draft the manuscript. All authors read and approved the final manuscript.

\section{Authors' information}

Jianying Ma: PhD, MD, Associate Professor of Medicine/Cardiology, Department of Cardiology, Zhongshan hospital, Fudan University; Shanghai institute of Cardiovascular disease, Shanghai, China. Juying Qian: MD, FSCAl, Professor of Medicine/Cardiology, Deputy Director of Department of Cardiology, Zhongshan hospital, Fudan University; Shanghai institute of Cardiovascular disease, Shanghai, China. Shufu Chang: MD, Attending physician, Department of Cardiology, Zhongshan hospital, Fudan University; Shanghai institute of Cardiovascular disease, Shanghai, China. Zhangwei Chen: MD, Attending physician, Department of Cardiology, Zhongshan hospital, Fudan University; Shanghai institute of Cardiovascular disease, Shanghai, China, Shanghai, China. Hang Jin: PhD, MD, Associate professor of Radiology, Department of Radiology, Zhongshan hospital, Fudan University, Shanghai, China. Mengsu Zeng: PhD, MD, Professor of Radiology, Director of Department of Radiology, Zhongshan hospital, Fudan University, Shanghai, China. Yunzeng Zou: Professor of Institute of Biomedical Science; Professor of Medicine/Cardiology, Department of Cardiology, Zhongshan hospital, Fudan University; Shanghai institute of Cardiovascular disease, Shanghai, China. GE Junbo, MD, FESC, FACC, FSCAl, Professor of Medicine/Cardiology, Director of Department of Cardiology, Zhongshan Hospital, Fudan University; Director of Shanghai Institute of Cardiovascular Diseases, Shanghai, China.

\section{Acknowledgements}

This study was supported by: National Natural Science Foundation of China (No. 30901383).

\section{Author details}

${ }^{1}$ Department of Cardiology, Zhongshan Hospital, Fudan University, Shanghai 200032, China. ${ }^{2}$ Shanghai Institute of Cardiovascular Diseases, Shanghai 200032, China. ${ }^{3}$ Department of Radiology, Zhongshan Hospital, Fudan University, Shanghai 200032, China.

Received: 26 July 2013 Accepted: 22 January 2014

Published: 4 February 2014

\section{References}

1. Heusch G, Kleinbongard P, Böse D, Levkau B, Haude M, Schulz R, Erbel R: Coronary microembolization: from bedside to bench and back to bedside. Circulation 2009, 120:1822-1836.

2. Dörge H, Neumann T, Behrends M, Skyschally A, Schulz R, Kasper C, Erbel R, Heusch G: Perfusion-contraction mismatch with coronary microvascular obstruction: role of inflammation. Am J Physiol Heart Circ Physiol 2000, 279: $\mathrm{H} 2587-\mathrm{H} 2592$
3. Thielmann M, Dörge H, Martin C, Belosjorow S, Schwanke U, van de Sand A, Konietzka I, Büchert A, Krüger A, Schulz R, Heusch G: Myocardial dysfunction with coronary microembolization: signal transduction through a sequence of nitric oxide, tumor necrosis factor- $a$ and sphingosine. Circ Res 2002, 90:807-813.

4. Skyschally A, Haude M, Dörge $H$, Thielmann M, Duschin A, van de Sand A, Konietzka I, Büchert A, Aker S, Massoudy P, Schulz R, Erbel R, Heusch G: Glucocorticoid treatment prevents progressive myocardial dysfunction resulting from experimental coronary microembolization. Circulation 2004, 109:2337-2342.

5. Dörge H, Schulz R, Belosjorow S, Post H, van de Sand A, Konietzka I, Frede S, Hartung T, Vinten-Johansen J, Youker KA, Entman ML, Erbel R, Heusch G: Coronary microembolization: the role of TNF-alpha in contractile dysfunction. J Mol Cell Cardiol 2002, 34:51-62.

6. Heusch P, Skyschally A, Leineweber K, Haude M, Erbel R, Heusch G: The interaction of coronary microembolization and ischemic preconditioning: a third window of cardioprotection through TNF-alpha. Arch Med Sci 2007, 3:83-92.

7. Li S, Zhong S, Zeng K, Luo Y, Zhang F, Sun X, Chen L: Blockade of NF-KB by pyrrolidine dithiocarbamate attenuates myocardial inflammatory response and ventricular dysfunction following coronary microembolization induced by homologous microthrombi in rats. Basic Res Cardiol 2010, 105:139-150.

8. Ma J, Qian J, Jin H, Chen Z, Chang S, Yang S, Sun AJ, Zeng MS, Zou YZ, Ge JB: Acute hyperenhancement on delayed contrast-enhanced magnetic resonance imaging is the characteristic sign after coronary microembolization. Chin Med J 2009, 122:687-691.

9. Ma J, Qian J, Ge J, Zeng X, Sun A, Chang S, Chen Z, Zou Y: Changes in left ventricular ejection fraction and coronary flow reserve after coronary microembolization. Arch Med Sci 2012, 8:63-69.

10. Breuckmann F, Nassenstein K, Bucher C, Konietzka I, Kaiser G, Konorza T, Naber C, Skyschally A, Gres P, Heusch G, Erbel R, Barkhausen J: Systematic analysis of functional and structural changes after coronary microembolization: a cardiac magnetic resonance imaging study. J Am Coll Cardiol Img 2009, 2:121-130.

11. Carlsson M, Wilson M, Martin AJ, Saeed M: Myocardial microinfarction after coronary microembolization in swine: MR imaging characterization. Radiology 2009, 250:703-713.

12. Cranney GB, Lotan CS, Dean L, Baxley W, Bouchard A, Pohost GM: Left ventricular volume measurement using cardiac axis nuclear magnetic resonance imaging. Validation by calibrated ventricular angiography. Circulation 1990, 82:154-163.

13. Herrogods M, De Paep G, Bijnens B, Bogaert JG, Rademakers FE, Bosmans HT, Bellon EP, Marchal GJ, Baert AL, Van de Werf F, De Geest H: Determination of left ventricular volume by two-dimensional echocardiography: comparison with magnetic resonance imaging. Eur Heart J 1994, 15:1070-1073.

14. Falkenstein E, Tillmann H-C, Christ M, Feuring M, Wehling M: Multiple actions of steroid hormones: a focus on rapid, nongenomic effects. Pharmacol Rev 2000, 52:513-555.

15. Giugliano GR, Giugliano RP, Gibson CM, Kuntz RE: Meta-analysis of corticosteroid treatment in acute myocardial infarction. Am J Cardiol 2003, 91:1055-1059.

16. Otto S, Seeber M, Fujita B, Kretzschmar D, Ferrari M, Goebel B, Figulla HR, Poerner TC: Microembolization and myonecrosis during elective percutaneous coronary interventions in diabetic patients: an intracoronary Doppler ultrasound study with 2-year clinical follow-up. Basic Res Cardiol 2012, 107:289.

17. Falk E: Unstable angina with fatal outcome: Dynamic coronary thrombosis leading to infarction and/or sudden death: Autopsy evidence of recurrent mural thrombosis with peripheral embolization culminating in total vascular occlusion. Circulation 1985, 71:699-708.

18. Davies MJ, Thomas AC, Knapman PA, Hangartner JR: Intramyocardial platelet aggregation in patients with unstable angina suffering sudden ischemic cardiac death. Circulation 1986, 73:418-427.

19. Topol EJ, Yadav JS: Recognition of the importance of embolization in atherosclerotic vascular disease. Circulation 2000, 101:570-580

20. Skyschally A, Schulz R, Gres P, Konietzka I, Martin C, Haude M, Erbel R, Heusch G: Coronary microembolization does not induce acute preconditioning against infarction in pigs - the role of adenosine. Cardiovasc Res 2004, 63:313-322.

21. Irwin MW, Mak S, Mann DL, Qu R, Penninger JM, Yan A, Dawood F, Wen WH, Shou Z, Liu P: Tissue expression and immunolocalization of tumor necrosis factor-a in postinfarction dysfunctional myocardium. Circulation 1999, 99:1492-1498. 
22. Hori M, Gotoh $K$, Kitakaze M, Iwai K, I wakura K, Sato H, Koretsune $\mathrm{Y}$, Inoue $\mathrm{M}$, Kitabatake A, Kamada T: Role of oxygen-derived free radicals in myocardial edema and ischemia in coronary microvascular embolization. Circulation 1991, 84:828-840.

23. Canton M, Skyschally A, Menabò R, Boengler K, Gres P, Schulz R, Haude M, Erbel R, Di Lisa F, Heusch G: Oxidative modification of tropomyosin and myocardial dysfunction following coronary microembolization. Eur Heart J 2006, 27:875-881.

24. Cronstein BN, Kimmel SC, Levin Rl, Martiniuk F, Weissmann G: A mechanism for the anti-inflammatory effects of corticosteroids: the glucocorticoid receptor regulates leukocyte adhesion to endothelial cells and expression of endothelial-leukocyte adhesion molecule 1 and intercellular adhesion molecule 1. Proc Natl Acad Sci U S A 1992, 89:9991-9995.

25. Williams CM, Coleman JW: Induced expression of mRNA for IL-5, IL-6, TNF-alpha, MIP-2 and IFN-gamma in immunologically activated rat peritoneal mast cells: inhibition by dexamethasone and cyclosporin A. Immunology 1995, 86:244-249.

26. Arras M, Strasser R, Mohri M, Doll R, Eckert P, Schaper W, Schaper J: Tumor necrosis factor-alpha is expressed by monocytes/macrophages following cardiac microembolization and is antagonized by cyclosporine. Basic Res Cardiol 1998, 93:97-107.

27. Henning RJ, Becker H, Vincent JL, Thijs L, Kalter E, Weil MH: Use of methylprednisolone in patients following acute myocardial infarction. Hemodynamic and metabolic effects. Chest 1981, 79:186-194.

doi:10.1186/2047-783X-19-7

Cite this article as: Ma et al:: Left ventricular remodeling with preserved function after coronary microembolization: the effect of methylprednisolone. European Journal of Medical Research 2014 19:7.

\section{Submit your next manuscript to BioMed Central and take full advantage of:}

- Convenient online submission

- Thorough peer review

- No space constraints or color figure charges

- Immediate publication on acceptance

- Inclusion in PubMed, CAS, Scopus and Google Scholar

- Research which is freely available for redistribution 